Punjab University Journal of Mathematics (2021),53(9),606-620

https://doi.org/10.52280/pujm.2021.530901

\title{
On Properties of $\alpha$-Sumudu Transform and Applications
}

\author{
J.A.Nanware \\ Department of Mathematics, \\ Shrikrishna Mahavidyalaya, Gunjoti \\ Dt. Osmanabad (M.S) - 413 606, India \\ Email: jag_skmg91@ @rediffmail.com \\ N.G.Patil \\ Department of Applied Sciences, \\ MBES College of Engineering, Ambajogai \\ Dt.Beed (M.S)- 413 517, India \\ Email: ngpatil1608@gmail.com
}

Received: 26 June, 2020 / Accepted: 09 August, 2021 / Published online: 25 September, 2021

\begin{abstract}
The $\alpha$-Sumudu transform is defined and its properties are proved. $\alpha$-Sumudu transform of convolution product and composition of functions is obtained. The $\alpha$-Sumudu transform of Riemann-Liouville integral and derivatives of fractional order are determined. As an application, the solution of Initial Value Problems with Riemann-Liouville derivative of fractional order is obtained. .
\end{abstract}

\section{AMS (MOS) Subject Classification Codes: 34A12; 26A33;44A35}

Key Words: Riemann-Liouville derivative, Sumudu transform, Initial value problems, Mittag-Leffler functions.

\section{INTRODUCTION}

Grunwald, Letnikov, Riemann-Liouville, Caputo, Miller and Ross, Hadamard [29] and Jumarie etc. types of fractional derivatives were introduced. Many natural phenomena are modeled via fractional differential equations. The concept of fractional calculus was defined in $17^{\text {th }}$ century. Researchers have found and studied several methods for obtaining analytical and approximate solution of fractional differential equations which includes Power series method [2, 13, 27, 26], Iterative method, Monotone iterative method, Homotopy Perturbation method [10], Adomian method [28] and Transforms methods [5, 8] etc. Integral transform methods [31] like New transform [30], Laplace transform [11], Sumudu transform [8], and Natural Transform [6] etc. are applied to study the solutions of differential equations of arbitrary order [16]. The Laplace integral transforms of Mathematical physics with the general scheme for applications was illustrated by Luchko [23]. Analytical solutions of some fractional ordinary differential equations are studied by Bulut et.al.[9] 
and applied Sumudu transform technique and found that this technique is direct and valuable to fractional differential equations. Analytical solution of fractional model of HIV infection of $C D 4^{+} T$ lymphocite cells was obtained by Bulut et.al.[10] using HPSTM and HATM. It is observed that HATM method is rapid and gives large convergence region by choosing appropriate value of $h$. Comparative study of fractional models using NDTM and VIM was carried in [7]. Transport models are governed by fractional partial differential equations and are investigated by researchers via fractional Sumudu transform [22], Walsh function [19, 21] and Chebyshev polynomials [20]. Linear and nonlinear partial differential equations using natural transform decomposition method was also studied in [6]. The Conformable fractional Laplace and Sumudu transforms were studied by Hammed et. al. $[17,1]$. Hammed et.al. introduced conformable fractional derivative and study its properties. Recently, Al-Zhour et.al.[3] studied fractional differential equations in conformable fractional derivative and obtained series solution for Laguere and Lane-Emden fractional differential equations and nonlinear dispersive PDEs [12, 27]. The conformable fractional natural transform have been studied by Al-Zhour et.al.[4] and applied to obtain solution of fractional differential equations. New technique is introduced by El-Ajou et. al.[14] to obtain solution of non-homogeneous higher order matrix fractional differential equations. Numerical solution of the fractional multi-pantagraph system is studied by El-Ajou et.al. [15] using algorithm of HAM and RPSM.

The $\alpha$ - Laplace transform firstly introduced by Romero et. al.[24] and applied to obtain solution of fractional differential equations. Medina et. al. [25] also applied the idea of $\alpha$-Laplace transform to find the solution of differential equations of $\alpha$ order [24]. This motivates us to define the $\alpha$-Sumudu transform and we apply this to find the solution of differential equations of fractional order.

In this paper, the $\alpha$-Sumudu transform is defined and fundamental properties of $\alpha$ Sumudu transform are obtained. The $\alpha$-Sumudu transform of a Riemann-Liouville integral and derivative of fractional order, Mittag-Leffler functions, convolution of two functions are determined. The $\alpha$-Sumudu transform is applied to obtain solution of initial value problems involving R-L fractional derivative.

The paper is compiled as under: In section 2, basic definitions and results are considered. We define $\alpha$-Sumudu transform, prove its properties, the inverse $\alpha$-Sumudu transform, convolution product, $\alpha$-Sumudu transform composition and $\alpha$-Sumudu transform of R-L derivative are given in third section. As an application of the $\alpha$-Sumudu transform, solution of initial value problem is obtained in section 4. Concluding remarks are given at the end.

\section{PRELIMINARIES}

In this section, we consider basic definitions and results in fractional calculus that are required in further section.

Definition 2.1. [29] The Gamma function $\Gamma(z)$ due to Euler is defined as

$$
\Gamma(z)=\int_{0}^{\infty} e^{-p} t^{z-1} d p ; \quad R(z)>0
$$


Definition 2.2. [29] Mittag-Leffler (M-L) function with 1-parameter is defined as

$$
E_{\alpha}(z)=\sum_{p=0}^{\infty} \frac{z^{p}}{\Gamma(\alpha p+1)}
$$

Definition 2.3. [29] M-L function with 2-parameter is defined as

$$
E_{\alpha, \beta}(z)=\sum_{p=0}^{\infty} \frac{z^{p}}{\Gamma(\alpha p+\beta)}
$$

Mittag-Leffler functions of $\lambda t^{\alpha}$ are

$$
E_{\alpha}\left(\lambda t^{\alpha}\right)=\sum_{p=0}^{\infty} \frac{\left(\lambda t^{\alpha}\right)^{p}}{\Gamma(\alpha p+1)}, \quad E_{\alpha, \beta}\left(\lambda t^{\alpha}\right)=\sum_{p=0}^{\infty} \frac{\left(\lambda t^{\alpha}\right)^{p}}{\Gamma(\alpha p+\beta)}
$$

and $m^{\text {th }}$ power of these functions is as follows:

$$
\begin{aligned}
E_{\alpha}^{m}\left(\lambda t^{\alpha}\right) & =\sum_{p=0}^{\infty} \frac{(p+m) !}{p !} \frac{\left(\lambda t^{\alpha}\right)^{p}}{\Gamma(\alpha p+\alpha m+1)} \\
E_{\alpha, \beta}^{m}\left(\lambda t^{\alpha}\right) & =\sum_{p=0}^{\infty} \frac{(p+m) !}{p !} \frac{\left(\lambda t^{\alpha}\right)^{p}}{\Gamma(\alpha p+\alpha m+\beta)}
\end{aligned}
$$

Definition 2.4. If $f(t)$ be defined in a interval $[a, \infty)$ then we say that $f(t)$ is locally integrable in $[a, \infty)$ if for all $a<b, f$ is integrable in $[a, b]$.

Definition 2.5. A real valued function $f(t)$ is said to be measurable if, for each $\eta \in R$, the set $[t: f(t)>\eta]$ is measurable.

Definition 2.6. [29] If $f(t)$ is locally integrable on $[a, \infty)$, then $R$-L integral of fractional order $\alpha$, is

$$
I^{\alpha} f(t)=\frac{1}{\Gamma(\alpha)} \int_{a}^{t}(t-p)^{\alpha-1} f(p) d p ; \quad t>a ; R(\alpha)>0
$$

Similarly, if $f(t)$ is locally integrable on $[-\infty, b)$ then

$$
I_{b}^{\alpha} f(t)=\frac{1}{\Gamma(\alpha)} \int_{t}^{b}(p-t)^{\alpha-1} f(p) d p ; \quad R(\alpha)>0
$$

Definition 2.7. [29] Riemann-Liouville ( $R-L)$ derivative of fractional order $\alpha$, is defined as

$$
D_{p}^{\alpha} f(p)=\left(\frac{d}{d p}\right)^{m} I_{p}^{m-\alpha} f(p) ; \quad R(\alpha)>0, \quad m \in I
$$

Definition 2.8. [31] The Sumudu transform of $f(t), \quad t \geq 0$, denoted by $F(u)$ is defined as

$$
F(u)=S[f(t) ; u]=\int_{0}^{\infty} \frac{1}{u} e^{-\frac{t}{u}} f(t) d t ; \quad u \in R
$$


Definition 2.9. [31] Let $A\left(R_{0}^{+}\right)$be the function space of Sumudu transformable functions, that is

$$
A\left(R_{0}^{+}\right)=\left\{f(t)\left|\exists N, \mu_{1}, \mu_{2}>0,\right| f(t) \mid<N e^{\frac{|t|}{\mu_{j}}} \quad \text { if } \quad t \in(-1)^{j} \times[0, \infty)\right\}
$$

for function in $A\left(R_{0}^{+}\right)$, and $N<\infty, \quad \mu_{j}, j=1,2$, may be finite or infinite.

Definition 2.10. [31] If $f(t)$ be defined on $R_{0}^{+}$, then incomplete Sumudu transform $F(u)$ of $f(t)$ is defined as

$$
S[f(t), b](u)=\int_{0}^{b} \frac{1}{u} e^{-\frac{t}{u}} f(t) d t ; \quad \text { for } \quad b, u \in R
$$

Theorem 2.11 (Fubini's Theorem). If $f(x, y)$ is continuous function on a rectangle $R=[a, b] \times[c, d]$, then $\iint_{R} f(x, y) d A=\int_{c}^{d} \int_{a}^{b} f(x, y) d x d y=\int_{a}^{b} \int_{c}^{d} f(x, y) d y d x$.

Lemma 2.12. [18] If $f(t)$ is well-behaved (not violating any assumptions like continuity, differentiability etc.) and $\alpha \in(0,1)$, then Sumudu transform of $R$-L fractional integral of $f(u)$ is

$$
S\left[I^{\alpha} f\right](u)=u^{\alpha} S[f](u) ; \quad R(\alpha)>0
$$

Lemma 2.13. [8] If $f(t)$ is well-behaved and $\alpha \in(0,1)$, then Sumudu transform of $R-L$ fractional derivative of $f(t)$ is

$$
S\left[D^{\alpha} f(t)\right](u)=u^{-\alpha} S[f(t)](u)-\left.\frac{I^{1-\alpha} f(t)}{u}\right|_{t=0}
$$

\section{3. $\alpha$-Sumudu TRANSFORM AND PROPERTIES}

Here, we define $\alpha$-Sumudu transform, convolution product and study its properties. So far in the literature $\alpha$-Sumudu transform is not defined yet.

Definition 3.1. If $f(t)$ is defined on $R_{0}^{+}$, then the $\alpha$-Sumudu transform $F_{\alpha}(u)$ is

$$
F_{\alpha}(u)=S_{\alpha}[f(t)](u)=\int_{0}^{\infty} \frac{1}{u^{\frac{1}{\alpha}}} e^{-\frac{t}{u^{\frac{1}{\alpha}}}} f(t) d t ; \quad u \in R .
$$

The $\alpha$-Sumudu transform is a generalization of Sumudu transform because as $\alpha \rightarrow 1$, we have

Thus, we have

$$
S_{1}[f(p)](u)=S[f(p)](u) .
$$

Theorem 3.2. If $f(t) \in A\left(R_{0}^{+}\right)$, then $F_{\alpha}(u)=S_{\alpha}[f(t)](u)$ for $u>a^{\alpha}$

Proof. It is obvious from the definition of $\alpha$-Sumudu transform.

Definition 3.3. [24] If $f, g \in L^{1}\left(R^{+}\right)$(measurable space), then classical convolution product is

$$
(f * g)(t)=\int_{0}^{t} f(\mu) g(t-\mu) d \mu, \quad t>0,
$$

where $L^{1}\left(R^{+}\right)=\left\{f: R \rightarrow C \mid\right.$ fis measurable and $\left.\int|f(t)| d t<\infty\right\}$. 
Definition 3.4. [25] If $f, g \in L^{1}\left(R^{+}\right)$, then convolution product o is defined as

$$
(f \circ g)(t)=\int_{t}^{\infty} f(\mu-t) g(\mu) d \mu, \quad t>0
$$

Lemma 3.5. If $f$ is well-behaved and $\alpha \in(0,1)$, then the $\alpha$-Sumudu transform of $f(u)$ is

$$
S_{\alpha}[f](u)=S[f](\mu) ; \mu=u^{\frac{1}{\alpha}}
$$

Proof. By $\alpha$-Sumudu transform

$$
\begin{aligned}
S_{\alpha}[f(t)](u) & =\int_{0}^{\infty} \frac{1}{u^{\frac{1}{\alpha}}} e^{-\frac{t}{u^{\frac{1}{\alpha}}}} f(t) d t ; \quad u \in R \\
& =S[f(t)] u^{\frac{1}{\alpha}} \\
& =S[f(t)] \mu, \quad \text { where } \quad \mu=u^{\frac{1}{\alpha}} \\
& =S[f] \mu ; \quad \mu=u^{\frac{1}{\alpha}}
\end{aligned}
$$

Theorem 3.6. If $a, c \in R$ and $0<\alpha \leq 1$, then
(a) $S_{\alpha}[c]=c$
(b) $S_{\alpha}\left[e^{a t}\right]=\frac{1}{1-a u^{\frac{1}{\alpha}}}$
(c) $S_{\alpha}[\sin a t]=\frac{a u^{\frac{1}{\alpha}}}{1+a^{2} u^{\frac{2}{\alpha}}}$
(d) $S_{\alpha}[$ cosat $]=\frac{1}{1+a^{2} u^{\frac{2}{\alpha}}}$
(e) $S_{\alpha}[\sinh a t]=\frac{a u^{\frac{1}{\alpha}}}{1-a^{2} u^{\frac{2}{\alpha}}}$
(f) $S_{\alpha}[\cosh a t]=\frac{1}{1-a^{2} u^{\frac{2}{\alpha}}}$
(g) $S_{\alpha}\left[t^{n}\right]=n ! u^{\frac{n}{\alpha}}$

Proof. By Definition 3.1, we have
(a) $S_{\alpha}[c]=\int_{0}^{\infty} \frac{1}{u^{\frac{1}{\alpha}}} e^{-\frac{t}{u^{\frac{1}{\alpha}}}} c d t=\frac{c}{u^{\frac{1}{\alpha}}}\left[\frac{e^{-\frac{t}{u^{\frac{1}{\alpha}}}}}{-\frac{1}{u^{\frac{1}{\alpha}}}}\right]_{0}^{\infty}=c$
(b) $S_{\alpha}\left[e^{a t}\right]=\int_{0}^{\infty} \frac{1}{u^{\frac{1}{\alpha}}} e^{-\frac{t}{u^{\frac{1}{\alpha}}}} e^{a t} d t=\frac{1}{u^{\frac{1}{\alpha}}}\left[\frac{e^{-\left(\frac{1}{u^{\frac{1}{\alpha}}}-a\right) t}}{-\left(\frac{1}{u^{\frac{1}{\alpha}}}-a\right)}\right]_{0}^{\infty}=\frac{1}{1-a u^{\frac{1}{\alpha}}}$
(c) $S_{\alpha}[\sin (a t)]=\int_{0}^{\infty} \frac{1}{u^{\frac{1}{\alpha}}} e^{-\frac{t}{u^{\frac{1}{\alpha}}}} \sin (a t) d t$

$$
\begin{aligned}
& =\frac{1}{u^{\frac{1}{\alpha}}}\left[\frac{e^{-\frac{t}{u^{\frac{1}{\alpha}}}}}{\left(-\frac{1}{u^{\frac{1}{\alpha}}}\right)^{2}+a^{2}}\left[-\frac{1}{u^{\frac{1}{\alpha}}} \sin (a t)-a \cos (a t)\right]\right]_{0}^{\infty} \\
& =\frac{1}{u^{\frac{1}{\alpha}}}\left[\frac{1}{\left(\frac{1}{u^{\frac{1}{\alpha}}}\right)^{2}+a^{2}}\right]=\frac{a u^{\frac{1}{\alpha}}}{1+a^{2} u^{\frac{2}{\alpha}}}
\end{aligned}
$$

(d) $S_{\alpha}[$ cosat $]=\int_{0}^{\infty} \frac{1}{u^{\frac{1}{\alpha}}} e^{-\frac{t}{u^{\frac{1}{\alpha}}}} \cos a t d t$

$$
=\frac{1}{u^{\frac{1}{\alpha}}}\left[\frac{e^{-\frac{t}{u^{\frac{1}{\alpha}}}}}{\left(-\frac{1}{u^{\frac{1}{\alpha}}}\right)^{2}+a^{2}}\left[-\frac{1}{u^{\frac{1}{\alpha}}} \cos a t+\text { asinat }\right]\right]_{0}^{\infty}=\frac{1}{u^{\frac{1}{\alpha}}}\left[\left(-\frac{1}{\left(\frac{1}{u^{\frac{1}{\alpha}}}\right)^{2}+a^{2}}\left(-\frac{1}{u^{\frac{1}{\alpha}}}\right)\right]=\right.
$$


$\frac{1}{1+a^{2} u^{\frac{2}{\alpha}}}$

Similarly, we can prove (e)and (f)

(g) $S_{\alpha}\left[t^{n}\right]=\int_{0}^{\infty} \frac{1}{u^{\frac{1}{\alpha}}} e^{-\frac{t}{u^{\frac{1}{\alpha}}}} t^{n} d t$

$$
\begin{aligned}
& \text { Let } \quad \frac{t}{u^{\frac{1}{\alpha}}}=x \quad \therefore t=x u^{\frac{1}{\alpha}} ; \quad \therefore d t=u^{\frac{1}{\alpha}} d x \\
& S_{\alpha}\left[t^{n}\right]=\int_{0}^{\infty} \frac{1}{u^{\frac{1}{\alpha}}} e^{-x} x^{n} u^{\frac{n}{\alpha}} u^{\frac{1}{\alpha}} d x=u^{\frac{n}{\alpha}} \int_{0}^{\infty} e^{-x} x^{n} d x=u^{\frac{n}{\alpha}} \Gamma(n+1)=n ! u^{\frac{n}{\alpha}}
\end{aligned}
$$

Theorem 3.7. Let $f, g:[0, \infty) \rightarrow R, \quad \lambda, \mu \in R$ and $0<\alpha \leq 1$. If $S_{\alpha}[f(t)]=$ $F_{\alpha}[u], \quad S_{\alpha}[g(t)]=G_{\alpha}[u]$, then

(i)

$$
S_{\alpha}[\lambda f(t)+\mu g(t)]=\lambda F_{\alpha}(u)+\mu G_{\alpha}(u)
$$

(ii)

(iii)

$$
S_{\alpha}\left[e^{-a t} f(t)\right]=F_{\alpha}\left[\frac{1}{u^{\frac{1}{\alpha}}}+a\right]
$$

$$
S_{\alpha}\left[f^{\prime}(t)\right]=\frac{1}{u^{\frac{1}{\alpha}}} F_{\alpha}[u]-\frac{1}{u^{\frac{1}{\alpha}}} f(0)
$$

(iv)

$$
S_{\alpha}\left[\int_{0}^{t} f(t) d t\right]=u^{\frac{1}{\alpha}} F_{\alpha}[u]
$$

Proof. By $\alpha$-Sumudu transform :

(i)

$$
\begin{aligned}
S_{\alpha}[\lambda f(t)+\mu g(t)] & =\int_{0}^{\infty} \frac{1}{u^{\frac{1}{\alpha}}} e^{-\frac{t}{u^{\frac{1}{\alpha}}}}[\lambda f(t)+\mu g(t)] d t \\
& =\lambda \int_{0}^{\infty} \frac{1}{u^{\frac{1}{\alpha}}} e^{-\frac{t}{u^{\frac{1}{\alpha}}}} f(t) d t+\mu \int_{0}^{\infty} \frac{1}{u^{\frac{1}{\alpha}}} e^{-\frac{t}{u^{\frac{1}{\alpha}}}} g(t) d t \\
& =\lambda F_{\alpha}[u]+\mu G_{\alpha}[u]
\end{aligned}
$$

(ii)

$$
\begin{aligned}
S_{\alpha}\left[e^{-a t} f(t)\right] & =\int_{0}^{\infty} \frac{1}{u^{\frac{1}{\alpha}}} e^{-\frac{t}{u^{\frac{1}{\alpha}}}} e^{-a t} f(t) d t \\
& =\int_{0}^{\infty} \frac{1}{u^{\frac{1}{\alpha}}} e^{-\left(\frac{1}{u^{\frac{1}{\alpha}}}+a\right) t} f(t) d t \\
& =F_{\alpha}\left[\frac{1}{u^{\frac{1}{\alpha}}}+a\right]
\end{aligned}
$$

Similarly, we prove

$$
S_{\alpha}\left[e^{a t} f(t)\right]=F_{\alpha}\left[\frac{1}{u^{\frac{1}{\alpha}}}-a\right]
$$


(iii)

$$
\begin{aligned}
S_{\alpha}\left[f^{\prime}(t)\right] & =\int_{0}^{\infty} \frac{1}{u^{\frac{1}{\alpha}}} e^{-\frac{t}{u^{\frac{1}{\alpha}}}} f^{\prime}(t) d t \\
& =\left[\frac{1}{u^{\frac{1}{\alpha}}} e^{-\frac{t}{u^{\frac{1}{\alpha}}}} f(t)\right]_{0}^{\infty}-\int_{0}^{\infty} \frac{1}{u^{\frac{1}{\alpha}}}\left(-\frac{1}{u^{\frac{1}{\alpha}}}\right) e^{-\frac{t}{u^{\frac{1}{\alpha}}}} f(t) d t \\
& =\left[0-\frac{1}{u^{\frac{1}{\alpha}}} f(0)\right]+\frac{1}{u^{\frac{1}{\alpha}}} \int_{0}^{\infty} \frac{1}{u^{\frac{1}{\alpha}}} e^{-\frac{t}{u^{\frac{1}{\alpha}}}} f(t) d t \\
& =\frac{1}{u^{\frac{1}{\alpha}}} F_{\alpha}[u]-\frac{1}{u^{\frac{1}{\alpha}}} f(0)
\end{aligned}
$$

In general

$$
S_{\alpha}\left[f^{n}(t)\right]=\frac{1}{u^{\frac{n}{\alpha}}} F_{\alpha}[u]-\frac{1}{u^{\frac{n}{\alpha}}} f(0)-\frac{1}{u^{\frac{n-1}{\alpha}}} f^{\prime}(0)-\frac{1}{u^{\frac{n-2}{\alpha}}} f^{\prime \prime}(0) \ldots-f^{n-1}(0) .
$$

(iv) Let $\phi(t)=\int_{0}^{t} f(t) d t, \quad \phi(0)=0 \quad \phi^{\prime}(t)=f(t)$

$$
\begin{aligned}
S_{\alpha}\left[\phi^{\prime}(t)\right] & =\frac{1}{u^{\frac{1}{\alpha}}} S_{\alpha}[\phi(t)]-\frac{1}{u^{\frac{1}{\alpha}}} \phi(0) \\
& =\frac{1}{u^{\frac{1}{\alpha}}} S_{\alpha}[\phi(t)](\because \phi(0)=0) \\
S_{\alpha}[\phi(t)] & =u^{\frac{1}{\alpha}} S_{\alpha}\left[\phi^{\prime}(t)\right]
\end{aligned}
$$

Putting the value of $\phi(t)$ and $\phi^{\prime}(t)$, to obtain

$$
S_{\alpha}\left[\int_{0}^{t} f(t) d t\right]=u^{\frac{1}{\alpha}} F_{\alpha}[u]
$$

Theorem 3.8. For $f^{(k)}(t) \in A\left(R_{0}^{+}\right), k=1,2 \ldots n$, we have

$$
S_{\alpha}\left[\left(\frac{d}{d t} f(t)\right)^{n}\right](u)=\frac{1}{u^{\frac{n}{\alpha}}} S_{\alpha}[f(t)](u)-\frac{1}{u^{\frac{n}{\alpha}}} \sum_{k=1}^{n} u^{\frac{n-k}{\alpha}} f^{\frac{n-k}{\alpha}}(0)
$$

Proof. Using

$$
\begin{gathered}
S\left[\left(\frac{d}{d t} f(t)\right)^{n}\right](\mu)=\frac{1}{\mu^{n}} S_{\alpha}[f(t)](u)-\frac{1}{\mu^{n}} \sum_{k=1}^{n} \mu^{n-k} f^{n-k}(0) \\
S_{\alpha}[f](u)=S[f](\mu) ; \quad \mu=u^{\frac{1}{\alpha}}
\end{gathered}
$$

we obtain

$$
S_{\alpha}\left[\left(\frac{d}{d t} f(t)\right)^{n}\right](u)=\frac{1}{u^{\frac{n}{\alpha}}} F_{\alpha}(u)-\frac{1}{u^{\frac{n}{\alpha}}} \sum_{k=1}^{n} u^{\frac{n-k}{\alpha}} f^{\frac{n-k}{\alpha}}(0)
$$

This proves the Theorem. 
Now, we turn to inversion formula

$$
S_{\alpha}[f](u)=S[f](\mu)=g_{1}(\mu) \quad \mu=u^{\frac{1}{\alpha}}
$$

then

$$
f(t)=S_{\alpha}^{-1}\left[S_{\alpha}[f](u)\right]=S^{-1}\left[g_{1}(\mu)\right](t)
$$

Applying inverse Sumudu transform, to obtain

$$
\begin{gathered}
S^{-1}\left[g_{1}(\mu)\right](t)=\frac{1}{2 \pi i} \int_{a-i \infty}^{a+i \infty} e^{\frac{t}{\mu}} g_{1}(\mu) d \mu \\
S^{-1}\left[g_{1}(\mu)\right](t)=\frac{1}{2 \pi i} \int_{a-i \infty}^{a+i \infty} e^{\frac{t}{\mu}} S[f](\mu) d \mu
\end{gathered}
$$

Change of variable $\mu=u^{\frac{1}{\alpha}} ; d \mu=\frac{1}{\alpha} u^{\frac{1}{\alpha}-1} d u$ gives

$$
S^{-1}\left[g_{1}(\mu)\right](t)=\frac{1}{2 \pi i} \int_{a^{\alpha}-i \infty}^{a^{\alpha}+i \infty} e^{\frac{t}{u^{\frac{1}{\alpha}}}} S_{\alpha}[f](u) \frac{1}{\alpha} u^{\frac{1}{\alpha}-1} d u
$$

Thus, we have

Definition 3.9. If $f$ is well-behaved and $\alpha \in(0,1)$,then inverse $\alpha$-Sumudu transform is

$$
S^{-1}\left[F_{\alpha}(u)\right](t)=\frac{1}{2 \pi i \alpha} \int_{a^{\alpha}-i \infty}^{a^{\alpha}+i \infty} e^{\frac{t}{u^{\frac{1}{\alpha}}}} F_{\alpha}(u) u^{\frac{1}{\alpha}-1} d u
$$

It is easily seen that $S_{\alpha}\left[S_{\alpha}^{-1}\right]=I d$, by change of variable $\mu=u^{\frac{1}{\alpha}}$.

Theorem 3.10. If $f(t), g(t) \in A\left(R_{0}^{+}\right)$such that $F_{\alpha}(u)=S_{\alpha}[f(t)](u)$ and $G_{\alpha}(u)=$ $S_{\alpha}[g(t)](u)$ then $S_{\alpha}[f(t) * g(t)](u)=u^{\frac{1}{\alpha}} F_{\alpha}(u) G_{\alpha}(u)$

Proof. Use $\alpha$-Sumudu transform and convolution,to get

$$
\begin{aligned}
S_{\alpha}[f(t)](u) & =\int_{0}^{\infty} \frac{1}{u^{\frac{1}{\alpha}}} e^{-\frac{t}{u^{\frac{1}{\alpha}}}} f(t) d t \\
S_{\alpha}[(f * g)(t) ; u] & =\int_{0}^{\infty} \frac{1}{u^{\frac{1}{\alpha}}} e^{-\frac{t}{u^{\frac{1}{\alpha}}}}(f * g)(t) d t \\
& =\frac{1}{u^{\frac{1}{\alpha}}} \int_{0}^{\infty} e^{-\frac{t}{u^{\frac{1}{\alpha}}}} \int_{0}^{t} f(\tau) g(t-\tau) d t d \tau
\end{aligned}
$$

Fubini's theorem gives

$$
S_{\alpha}[(f * g)(t)](u)=\frac{1}{u^{\frac{1}{\alpha}}} \int_{0}^{\infty} e^{-\frac{\tau}{u^{\frac{1}{\alpha}}}} f(\mu) d \mu \int_{\mu}^{\infty} e^{-\frac{(t-\mu)}{u^{\frac{1}{\alpha}}}} g(t-\mu) d t
$$

Let $t-\mu=z$ and extension of upper bound of integrals to $t \rightarrow \infty$, gives

$$
\begin{gathered}
S_{\alpha}[(f * g)(t) ; u]=\frac{1}{u^{\frac{1}{\alpha}}} \int_{0}^{\infty} e^{-\frac{\mu}{u^{\frac{1}{\alpha}}}} f(\mu) d \mu \int_{0}^{\infty} e^{-\frac{z}{u^{\frac{1}{\alpha}}}} g(z) d z \\
S_{\alpha}[(f * g)(t) ; u]=u^{\frac{1}{\alpha}}\left[F_{\alpha}(u) \cdot G_{\alpha}(u)\right]
\end{gathered}
$$


Theorem 3.11. If $f, g \in L^{1}\left(R_{0}^{+}\right), 0<\alpha<1$ then $S_{\alpha}[$ fog $](u)=S_{\alpha}[g](u) . S[f]\left(-u^{\frac{1}{\alpha}}\right)$

Proof. Using definition of $\alpha$-Sumudu transform and convolution product $o$, we obtain

$$
\begin{aligned}
S_{\alpha}[f(t)](u) & =\int_{0}^{\infty} \frac{1}{u^{\frac{1}{\alpha}}} e^{-\frac{t}{u^{\frac{1}{\alpha}}}} f(t) d t \\
S_{\alpha}[(f \circ g)(t) ; u] & =\int_{0}^{\infty} \frac{1}{u^{\frac{1}{\alpha}}} e^{-\frac{t}{u^{\frac{1}{\alpha}}}}(f \circ g)(t) d t \\
& =\frac{1}{u^{\frac{1}{\alpha}}} \int_{0}^{\infty} e^{-\frac{t}{u^{\frac{1}{\alpha}}}} \int_{t}^{\infty} f(\mu-t) g(\mu) d t d \mu
\end{aligned}
$$

Fubini's theorem implies

$$
S_{\alpha}[(f \circ g)(t) ; u]=\frac{1}{u^{\frac{1}{\alpha}}} \int_{0}^{\infty} e^{-\frac{\mu}{u^{\frac{1}{\alpha}}}} g(\mu) d \mu \int_{0}^{\mu} e^{-\frac{(t-\mu)}{u^{\frac{1}{\alpha}}}} f(\mu-t) d t
$$

Let $\mu-t=z$ and extension of upper bound of integrals gives

$$
\begin{aligned}
S_{\alpha}[(f \circ g)(t) ; u] & =\frac{1}{u^{\frac{1}{\alpha}}} \int_{0}^{\infty} e^{-\frac{\tau}{u^{\frac{1}{\alpha}}}} g(\tau) d \tau \int_{\tau}^{0} e^{\frac{z}{u^{\frac{1}{\alpha}}}} f(z)(-d z) \\
& =\frac{1}{u^{\frac{1}{\alpha}}} \int_{0}^{\infty} e^{-\frac{\tau}{u^{\frac{1}{\alpha}}}} g(\tau) d \tau \int_{0}^{\tau} e^{\frac{z}{u^{\frac{1}{\alpha}}}} f(z)(d z) \\
S_{\alpha}[(f \circ g)(t) ; u] & =S_{\alpha}[g](u) . S[f]\left(-u^{\frac{1}{\alpha}}\right)
\end{aligned}
$$

Theorem 3.12. If $\lambda \in R^{+}$and $f, g \in L^{1}\left(R^{+}\right)$and $e_{\lambda^{\frac{1}{\alpha}}}(t)=e^{\frac{t}{\lambda^{\frac{1}{\alpha}}}} ; t \geq 0, t \in R^{+}$, then

(i) $f o \frac{1}{\lambda^{\frac{1}{\alpha}}} e_{\lambda^{\frac{1}{\alpha}}}=S_{\alpha}[f](\lambda) e_{\lambda^{\frac{1}{\alpha}}}$

(ii) $\frac{1}{\lambda^{\frac{1}{\alpha}}} e_{\lambda^{\frac{1}{\alpha}}}$ of $=S_{\alpha}[f]\left(\lambda^{\alpha}\right) e_{-\lambda^{\frac{1}{\alpha}}}-\left(e_{-\lambda} * f\right)$

Proof. $\quad$ (i) By definition 3.4, we have

$$
\left(f o \frac{1}{\lambda^{\frac{1}{\alpha}}} e_{\lambda^{\frac{1}{\alpha}}}\right)(t)=\int_{t}^{\infty} f(\mu-t) \frac{1}{\lambda^{\frac{1}{\alpha}}} e^{-\frac{\mu}{\lambda^{\frac{1}{\alpha}}}} d \mu
$$

If $z=\mu-t \quad$ then $d p=d \mu$

$$
\begin{aligned}
\left(f o \frac{1}{\lambda^{\frac{1}{\alpha}}} e_{\lambda^{\frac{1}{\alpha}}}\right)(t) & =\int_{0}^{\infty} f(z) \frac{1}{\lambda^{\frac{1}{\alpha}}} e^{-\frac{(z+t)}{\lambda^{\frac{1}{\alpha}}}} d z \\
& =\left[\int_{0}^{\infty} \frac{1}{\lambda^{\frac{1}{\alpha}}} e^{-\frac{z}{\lambda^{\frac{1}{\alpha}}}} d z\right] e_{\lambda^{\frac{1}{\alpha}}} \\
& =S_{\alpha}[f](\lambda) e_{\lambda^{\frac{1}{\alpha}}} .
\end{aligned}
$$


(ii) By definition 3.4, we have

$$
\left(\frac{1}{\lambda^{\frac{1}{\alpha}}} e_{\lambda^{\frac{1}{\alpha}}} o f\right)(t)=\int_{t}^{\infty} \frac{1}{\lambda^{\frac{1}{\alpha}}} e^{-\frac{(\mu-t)}{\lambda^{\frac{1}{\alpha}}}} f(\mu) d \mu
$$

as $f, y, e_{-\lambda^{\frac{1}{\alpha}}} \in L^{1}\left(R^{+}\right)$then $e_{-\lambda^{\frac{1}{\alpha}}} * f \in L^{1}\left(R^{+}\right)$, we obtain

$$
\begin{aligned}
\left(\frac{1}{\lambda^{\frac{1}{\alpha}}} e_{\lambda^{\frac{1}{\alpha}}} o f\right)(t) & =\left(\int_{0}^{\infty} \frac{1}{\lambda^{\frac{1}{\alpha}}} e^{-\frac{(\tau-t)}{\lambda^{\frac{1}{\alpha}}}} f(\tau) d \tau\right)\left(e_{-\lambda^{\frac{1}{\alpha}}} * f\right)(t) \\
& =\left(\int_{0}^{\infty} \frac{1}{\lambda^{\frac{1}{\alpha}}} e^{-\frac{(\tau)}{\lambda^{\frac{1}{\alpha}}}} f(\tau) d \tau\right) e_{-\lambda^{\frac{1}{\alpha}}}-\left(e_{-\lambda^{\frac{1}{\alpha}}} * f\right)(t) \\
& =S_{\alpha}[f](\lambda) e_{-\lambda^{\frac{1}{\alpha}}}-\left(e_{-\lambda^{\frac{1}{\alpha}}} * f\right)(t)
\end{aligned}
$$

Lemma 3.13. If $f$ is well-behaved and $\alpha \in(0,1)$, then $\alpha$-Sumudu transform of $R$ - $L$ fractional integral of $f$ is

$$
S_{\alpha}\left[I_{x}^{\gamma} f\right](u)=u^{\frac{\gamma}{\alpha}} S_{\alpha}[F](u)
$$

Proof. Recall that $t>0, \beta \in R$ for

$$
S_{\alpha}\left[t^{\gamma}\right]=\Gamma(\gamma+1) \quad u^{\frac{\gamma}{\alpha}}
$$

Since

$$
I_{x}^{\gamma} f(x)=J_{\gamma}(t) * f(t), \text { where } J_{\gamma}(t)=\frac{t^{\gamma-1}}{\Gamma(\gamma)}
$$

we have from 3.3

$$
S_{\alpha}\left[J_{\gamma}(t)\right](u)=S_{\alpha}\left[\frac{t^{\gamma-1}}{\Gamma \gamma}\right]=u^{\frac{\gamma-1}{\alpha}}
$$

By definition of $\alpha$-Sumudu transform and using Theorem 3.5, we obtain

$$
\begin{aligned}
S_{\alpha}\left[I_{x}^{\gamma} f(x)\right] & =S_{\alpha}\left[J_{\gamma}(t) * f(t)\right](u) \\
& =u^{\frac{1}{\alpha}} S_{\alpha}\left[J_{\gamma}(t)\right](u) \cdot S_{\alpha}[f](u) \\
& =u^{\frac{1}{\alpha}} u^{\frac{\gamma-1}{\alpha}} S_{\alpha}[f](u) \\
& =u^{\frac{\gamma}{\alpha}} S_{\alpha}[f](u)
\end{aligned}
$$

Lemma 3.14. If $\alpha \in(0,1)$, then $\alpha$-Sumudu transform of fractional $R$ - $L$ derivative of $f(t)$ is

$$
S_{\alpha}\left[D_{x}^{\gamma} f(t)\right](u)=u^{-\frac{\gamma}{\alpha}} S_{\alpha}[f(t)](u)-\left.\frac{I_{x}^{1-\gamma}}{u} f(t)\right|_{t=0}
$$


Proof. Consider

$$
\begin{aligned}
S_{\alpha}\left[D_{x}^{\gamma} f(t)\right](u) & =S_{\alpha}\left[\frac{d}{d x} I_{x}^{1-\gamma} f(t)\right](u) \\
& =u^{-\frac{1}{\alpha}} S_{\alpha}\left[I_{x}^{1-\gamma} f(t)\right](u)-\left.\frac{I_{x}^{1-\gamma}}{u} f(t)\right|_{t=0} \\
& =u^{-\frac{1}{\alpha}} u^{\frac{1-\gamma}{\alpha}} S_{\alpha}[f(t)](u)-\left.\frac{I_{x}^{1-\gamma}}{u} f(t)\right|_{t=0} \\
& =u^{-\frac{\gamma}{\alpha}} S_{\alpha}[f(t)](u)-\left.\frac{I_{x}^{1-\gamma}}{u} f(t)\right|_{t=0}
\end{aligned}
$$

Theorem 3.15. If $\gamma \in C, R(\gamma)>0, \lambda \in R$, then

$$
S_{\alpha}\left[t^{\gamma m} E_{\alpha}^{m}\left(\lambda t^{\gamma}\right)\right]=\frac{u^{\frac{-\gamma}{\alpha}} m !}{\left(u^{\frac{-\gamma}{\alpha}}-\lambda\right)^{m+1}}
$$

Proof. Since

$$
\sum_{p=0}^{\infty} \frac{(p+m) !}{p !} \cdot x^{p}=\frac{m !}{(1-x)^{m+1}}
$$

we have

$$
\begin{aligned}
S_{\alpha}\left[t^{\gamma m} E_{\alpha}^{m}\left(\lambda t^{\gamma}\right)\right] & =\sum_{p=0}^{\infty} \frac{(p+m) !}{p !} \lambda^{p} \cdot \frac{S_{\alpha}\left[t^{\gamma p+\gamma m}\right]}{\Gamma(\gamma p+\gamma m+1)} \\
& =\sum_{p=0}^{\infty} \frac{(p+m) !}{p !} \lambda^{p} \cdot \frac{\Gamma(\gamma p+\gamma m+1)}{\Gamma(\gamma p+\gamma m+1)} \cdot u^{\frac{\gamma p+\gamma m}{\alpha}} \\
& =\sum_{p=0}^{\infty} \frac{(p+m) !}{p !} \lambda^{p} \cdot u^{\frac{\gamma p+\gamma m}{\alpha}} \\
& =u^{\frac{\gamma m}{\alpha}} \cdot \frac{m !}{\left(1-\lambda u^{\frac{\gamma}{\alpha}}\right)^{m+1}} \\
& =\frac{u^{\frac{\gamma m}{\alpha}} m !}{\left(u^{\frac{\gamma}{\alpha}}\right)^{m+1}\left(u^{-\frac{\gamma}{\alpha}}-\lambda\right)^{m+1}} \\
& =u^{\frac{\gamma m}{\alpha}} \cdot u^{-\frac{\gamma m}{\alpha}} \cdot u^{-\frac{\gamma}{\alpha}} \cdot \frac{m !}{\left(u^{-\frac{\gamma}{\alpha}}-\lambda\right)^{m+1}} \\
& =\frac{u^{-\frac{\gamma}{\alpha}} m !}{\left(u^{-\frac{\gamma}{\alpha}}-\lambda\right)^{m+1}}
\end{aligned}
$$

Theorem 3.16. If $\eta, \beta \in C, \quad R(\eta)>0, \quad R(\beta)>0, \quad \lambda \in R$, then

$$
S_{\alpha}\left[t^{\eta m-\beta-1} E_{\eta, \beta}^{m}\left(\lambda t^{\eta}\right)\right]=\frac{u^{\frac{\beta-\eta-1}{\alpha}} m !}{\left(u^{\frac{-\eta}{\alpha}}-\lambda\right)^{m+1}}
$$


Proof. Since

$$
\sum_{p=0}^{\infty} \frac{(p+m) !}{p !} \cdot x^{p}=\frac{m !}{(1-x)^{m+1}}
$$

then

$$
\begin{aligned}
S_{\alpha}\left[t^{\eta m+\beta-1} E_{\eta, \beta}^{m}\left(\lambda t^{\eta}\right)\right] & =\sum_{p=0}^{\infty} \frac{(p+m) !}{p !} \lambda^{p} \cdot \frac{S_{\alpha}\left[t^{\eta p+\eta m+\beta-1}\right]}{\Gamma(\eta p+\eta m+\beta)} \\
& =\sum_{p=0}^{\infty} \frac{(p+m) !}{p !} \lambda^{p} \cdot \frac{\Gamma(\eta p+\eta m+\beta)}{\Gamma(\eta p+\eta m+\beta)} \cdot u^{\frac{\eta p+\eta m+\beta-1}{\alpha}} \\
& =\sum_{p=0}^{\infty} \frac{(p+m) !}{p !} \lambda^{p} \cdot u^{\frac{\eta p+\eta m+\beta-1}{\alpha}} \\
& =u^{\frac{\eta m+\beta-1}{\alpha}} \sum_{p=0}^{\infty} \frac{(p+m) !}{p !}\left(\lambda u^{\frac{\eta}{\alpha}}\right)^{p} \\
& =u^{\frac{\eta m+\beta-1}{\alpha}} \frac{m !}{\left(1-\lambda u^{\frac{\eta}{\alpha}}\right)^{m+1}} \\
& =u^{\frac{\eta m+\beta-1}{\alpha}} \frac{m !}{u^{\frac{\eta(m+1)}{\alpha}}\left(u^{-\frac{\eta}{\alpha}}\right)^{m+1}} \\
& =u^{\frac{\eta m+\beta-1}{\alpha}} \cdot u^{\frac{-\eta m-\eta}{\alpha}} \cdot \frac{m !}{\left(u^{-\frac{\eta}{\alpha}}-\lambda\right)^{m+1}} \\
& =u^{\frac{\eta m+\beta-1-\eta m-\eta}{\alpha}} \cdot \frac{m !}{\left(u^{-\frac{\eta}{\alpha}}-\lambda\right)^{m+1}} \\
& =u^{\frac{\beta-\eta-1}{\alpha}} \cdot \frac{m !}{\left(u^{-\frac{\eta}{\alpha}}-\lambda\right)^{m+1}}
\end{aligned}
$$

\section{Applications}

As an application of $\alpha$-Sumudu transform, we obtain solution of following differential equations involving R-L fractional derivative:

Example 4.1. Consider the following fractional differential equation

$$
D^{\alpha} f(t)+a f(t)=0 ;\left.\quad I^{\alpha}\right|_{t=0}=c .
$$


Applying $\alpha$-Sumudu transform, to obtain

$$
\begin{aligned}
\frac{1}{u^{\frac{1}{\alpha}}} S_{\alpha}[f(t)](u)-\left.\frac{I^{\alpha}}{u^{\frac{1}{\alpha}}} f(t)\right|_{t=0}+a S_{\alpha}[f(t)](u)=S_{\alpha}[f(t)](u)\left[\frac{1}{u^{\frac{1}{\alpha}}}+a\right] & =\frac{c}{u^{\frac{1}{\alpha}}} \\
S_{\alpha}[f(t)](u)\left[\frac{1+a u^{\frac{1}{\alpha}}}{u^{\frac{1}{\alpha}}}\right] & =\frac{c}{u^{\frac{1}{\alpha}}} \\
S_{\alpha}[f(t)](u) & =\frac{c}{1+a u^{\frac{1}{\alpha}}}
\end{aligned}
$$

Applying inverse $\alpha$-Sumudu transform, we obtain the solution of (4. 6 )

$$
\begin{aligned}
S_{\alpha}^{-1}\left[S_{\alpha}[f(t)]\right](u) & =S_{\alpha}^{-1}\left[\frac{c}{1+a u^{\frac{1}{\alpha}}}\right] \\
f(t) & =c t^{-\alpha} E_{\alpha, \alpha}\left(-a t^{\alpha}\right) .
\end{aligned}
$$

Example 4.2. Consider the following fractional differential equation

$$
D^{\alpha} f(t)-1=t^{2} ;\left.\quad I^{\alpha}\right|_{t=0}=2
$$

Applying $\alpha$-Sumudu transform, to obtain

$$
\begin{aligned}
\frac{1}{u^{\frac{1}{\alpha}}} S_{\alpha}[f(t)](u)-\left.\frac{I^{\alpha}}{u^{\frac{1}{\alpha}}} f(t)\right|_{t}-S_{\alpha}[1] & =S_{\alpha}\left[t^{2}\right] \\
\frac{1}{u^{\frac{1}{\alpha}}} S_{\alpha}[f(t)](u) & =2 ! u^{\frac{2}{\alpha}}+1+\frac{2}{u^{\frac{1}{\alpha}}} \\
S_{\alpha}[f(t)](u) & =2 ! u^{\frac{2}{\alpha}} u^{\frac{1}{\alpha}}+u^{\frac{1}{\alpha}}+2
\end{aligned}
$$

Applying inverse $\alpha$-Sumudu transform, we obtain the solution of (4. 7 )

$$
\begin{aligned}
S_{\alpha}^{-1}\left[S_{\alpha}[f(t)]\right](u) & =S_{\alpha}^{-1}\left[2 ! u^{\frac{3}{\alpha}}+u^{\frac{1}{\alpha}}+2\right] \\
f(t) & =\frac{1}{3} t^{3}+t+2
\end{aligned}
$$

Example 4.3. Consider the following fractional differential equation

$$
D^{\alpha} f(t)-1=\cos 2 t+t^{2} ;\left.\quad I^{\alpha}\right|_{t=0}=2
$$

Applying $\alpha$-Sumudu transform, to obtain

$$
\begin{aligned}
\frac{1}{u^{\frac{1}{\alpha}}} S_{\alpha}[f(t)](u)-\left.\frac{I^{\alpha}}{u^{\frac{1}{\alpha}}} f(t)\right|_{t}-S_{\alpha}[1] & =S_{\alpha}[\cos 2 t]+S_{\alpha}\left[t^{2}\right] \\
\frac{1}{u^{\frac{1}{\alpha}}} S_{\alpha}[f(t)](u) & =\frac{1}{1+4 u^{\frac{2}{\alpha}}}+2 ! u^{\frac{2}{\alpha}}+1+\frac{2}{u^{\frac{1}{\alpha}}} \\
S_{\alpha}[f(t)](u) & =\frac{u^{\frac{1}{\alpha}}}{1+4 u^{\frac{2}{\alpha}}}+2 ! u^{\frac{2}{\alpha}} u^{\frac{1}{\alpha}}+u^{\frac{1}{\alpha}}+2
\end{aligned}
$$


Applying inverse $\alpha$-Sumudu transform, we obtain the solution of (4. 8 )

$$
\begin{aligned}
S_{\alpha}^{-1}\left[S_{\alpha}[f(t)]\right](u) & =S_{\alpha}^{-1}\left[\frac{u^{\frac{1}{\alpha}}}{1+4 u^{\frac{2}{\alpha}}}+2 u^{\frac{3}{\alpha}}+u^{\frac{1}{\alpha}}+2\right] \\
f(t) & =\frac{1}{2} \sin 2 t+\frac{1}{3} t^{3}+t+2
\end{aligned}
$$

\section{CONCLUSION}

The $\alpha$-Sumudu transform for Sumudu transformable functions is defined. The properties of $\alpha$-Sumudu transform such as convolution product and composition are proved. The $\alpha$-Sumudu transform of elementary functions are also obtained. The $\alpha$-Sumudu transform of Riemann- Liouville fractional integral and derivative, Mittag-Leffler function with one parameter and two parameters are established. The inverse $\alpha$-Sumudu transform is defined. The $\alpha$-Sumudu transform and inverse $\alpha$-Sumudu transform are used to obtain solution of fractional differential equations with initial conditions.

\section{ACKNOWLEDGEMENTS}

Authors are grateful to Reviewer's for reading whole manuscript carefully and for their valuable comments that helps to improve the writing of this paper.

\section{REFERENCES}

[1] Z. Al-Zhour, F. Alrawajeh, N. Al-Mutairi and R. Alkhasawneh, New Results on the Conformable Fractional Sumudu Transform, Theories and Applications, Int. J. Anal. Appl., 17, No. 6(2019) 1019-1033.

[2] Z. Al-Zhour, N. Al-Mutairi, F. Alrawajeh, R. Alkhasawneh, Series Solutions for the Laguerre and LaneEmden Fractional Differential Equations in the Sense of Conformable Fractional Derivative, Alexandria Eng. J., 8, No.4 (2019) 1413-1420.

[3] Z. Al-Zhour, Fundamental fractional exponential matrix: New computational formulae and electrical applications, Int.J. Electron. Commun.(AEU), 129 (2021) 153557.

[4] Z. Al-Zhour, N. Al-Mutairi, F. Alrawajeh, R. Alkhasawneh, New Theoretical Results and Applications on Conformable Fractional Natural Transform, Ain Shams Eng. J., 21, No.1 (2021) 927-933.

[5] M. Baloch, S. Iqbal ,F. Sarwar and Abdul Rehman,The Relationship of Fractional Transform with Fractional Fourier,Mellin and Sumudu Transforms, American Scie. Research J. Engi., Tech. Sci.(ASRJETS), 55,No. 1(2019) 11-16.

[6] H. M. Baskonus,H.Bulut and Y.Pandir, The Natural Transform Decomposition Method for Linear and Nonlinear Partial Differential Equations, Math. Engi. Sci. Aero., 5, No.1(2014) 111-126.

[7] H.M.Baskonus and H. Bulut, A comparison between NTDM and VIM for modified Cammassa-Holm and Modified Degasperis-Procesi Equations, Nonl. Studies,22,No. 4(2015) 601-611.

[8] D.S. Bodkhe and S.K. Panchal, On Sumudu Transform of Fractional Derivatives and its Applications to Fractional Differential Equations, Asian J. Math. Computer Research, 11, No.1(2016) 69-77.

[9] H.Bulut,H.M.Baskonus and F.B.M.Belgacem, The Analytical Solution of Some Fractional Ordinary Differential Eqautions by the SUmudu Transform Method, Abs. Appl. Anal., Vol.2013, ArticleID 203875, 6 Pages, http://dx.doi.org/10.1155/2013/203875.

[10] H.Bulut, Devendra Kumar,Jagdev Singh, Ram Swroop and H.M.Baskonous, Analytic Study for Fractional Model of HIV Infection of CD4+T Lymphocyte Cells, Math. Natural Science, 2(2018) 33-43, doi: $10.22436 / \mathrm{mns} .02 .01 .04$

[11] L. Debnath, and D. Bhatta, Integral Transforms and Their Applications, Chapman and Hall/CRC, Taylor and Francis Group,New York; 2007. 
[12] A. El-Ajou, M. Oqielat, Z. Al-Zhour, S. Kumar and S. Momani, Solitary solutions for time-fractional nonlinear dispersive PDEs in the sense of conformable fractional derivative, Chaos, 29 (2019) 093102.

[13] A. El-Ajou, Z. Al-Zhour M. Oqielat and S. Momani, T. Hayat, Series Solutions of Nonlinear Conformable Fractional KdV-Burgers Equation with Some Applications, The Eur. Phys. J. Plus, 134 (2019), 402.

[14] A. El-Ajou, M. Oqielat, Z. Al-Zhour and S. Momani, A Class of Linear Non-Homogenous Higher Order Matrix Fractional Differential Equations: Analytical Solutions and New Technique, Fract. Calc. Appl. Anal., 23, No.2 (2020) 356-377.

[15] A. El-Ajou, M. Oqielat, Z. Al-Zhour, S. Momani, Analytical Numerical Solutions of the Fractional MultiPantograph System: Two Attractive Methods and Comparisons, Results Phys., 14 (2019) 102500.

[16] H. Eltayeb and A. Kilicman, A Note on The Sumudu Transforms and Di?erential Equations, Appl. Math.Sciences, 4,No. 22(2010) 1089-1098.

[17] M. Abu Hammad and R. Khalil, Conformable Fractional Heat Differential Equation, Int.J. Pure Appl. Math,94, No.2(2014) 215-221.

[18] Q. D.Katatbeha and Fethi Bin Muhammad Belgacem, Applications of the Sumudu Transform to Fractional Differential Equations, Nonl. Studies, 18, No. 1(2011) 99-112.

[19] A.Kadem, Solving Transport Equation via Walsh Function and Sumudu Transform, Apppl. Comput.Math.7, No. 1(2008) 107-118.

[20] A.Kadem and Analele, Solving the One-dimensional Neutron Transport Equation Using Chebyshev Polynomials and The Sumudu Transform, Universitatii Oradea Fasc.Matematica, Tom XII (2005) 153-171.

[21] A.Kadem, Analytic Investigations of the Walsh Functions combined with the Sumudu Transform and Applications to the Transport Equation, Asian-European J. Math., 4, No.2(2011) 199-215.

[22] A.Kadem, A. Kilicman, Note on Transport Equation and Fractional Sumudu Transform, Comp. Math. Appl. 62(2011) 2992-3003. https://doi.org/10.1016/s0304-0208(06)x8001-5

[23] Y. Luchko, Some Schemata for Applications of the Integral Transforms of Mathematicl physics, Mathematics, 7, No. 254 (2019); doi: 10.3390/math7030254.

[24] G. D. Medina and N.R. Ojeda, J.H. Pereira and L. G. Romero, Fractional Laplace Transform and Fractional Calculus, Int. Mathematical Forum, 12, No.20(2017) 991-1000.

[25] P.J. Miana, Convolution Products in $L^{1}\left(R^{+}\right)$, Integral Transforms and Fractional Calculus, Frac. Cal. Appl. Anal., 8, No.4(2005) 361-370.

[26] J.A. Nanware, Gunwant A. Birajdar, Methods of Solving Fractional Differential Equations of Order $\alpha(0<$ $\alpha<1<$ ), Bull. Marathwada Mathematical Soci.,15, No.2(2014) 40-53.

[27] M. Oqielat, A. El-Ajou, Z. Al-Zhour, R. Alkhasawneh, H. Alrabaiah, Series solutions for nonlinear timefractional Schrodinger equations: Comparisions between conformable and Caputo derivatives, Alexandria Eng. J., 59, No.4 (2020) 2101-2114.

[28] T. Patel and Ramakanta Meher, Adomian Decomposition Sumudu Transform Method for Solving a Solid and Porous Fin with Temperature Dependent Internal Heat Generation, SpringerPlus 5489 (2016) DOI 10.1186/s40064-016-2106-8.

[29] I. Podlubny, Fractional Differential Equations, Academic Press, United States, 1999.

[30] R. Saadeh, Ahmad Qazza and Aliaa Burqan, A New Integral Transform: ARA Transform and Its Properties and Applications, Symmetry 12(2020) 925.

[31] G.K. Watugala, Sumudu transform- A New Integral Transform to Solve Differential Equations and Control Engineering Problems, Int.J.Math.Educ.Sci.Technol. 24, No. 1(1993) 35-43. https://doi.org/10.1080/0020739930240105 